\title{
OPEN Averaging sleep spindle occurrence in dogs predicts learning performance better than single measures
}

\author{
Ivaylo Borislavov lotchev ${ }^{1,4}$, Vivien Reicher ${ }^{1,3,4}$, Enikő Kovács ${ }^{1,2,4}$, Tímea Kovács ${ }^{1}$, \\ Anna Kis ${ }^{2,5}$, Márta Gácsii, ${ }^{1,3}$ \& Enikö Kubinyi ${ }^{1,5}$
}

Although a positive link between sleep spindle occurrence and measures of post-sleep recall (learning success) is often reported for humans and replicated across species, the test-retest reliability of the effect is sometimes questioned. The largest to date study could not confirm the association, however methods for automatic spindle detection diverge in their estimates and vary between studies. Here we report that in dogs using the same detection method across different learning tasks is associated with observing a positive association between sleep spindle density (spindles/minute) and learning success. Our results suggest that reducing measurement error by averaging across measurements of density and learning can increase the visibility of this effect, implying that trait density (estimated through averaged occurrence) is a more reliable predictor of cognitive performance than estimates based on single measures.

Sleep spindles are thalamocortical transmissions ${ }^{1,2}$ observed mostly in mammalian non-REM sleep ${ }^{3}$ as brief $\left(0.5-5 \mathrm{~s}^{4}\right)$ trains of symmetric waves ${ }^{5}$ in the EEG signal. Different propositions for their defining frequency (waves/second) overlap in the $9-16 \mathrm{~Hz}$ band among humans ${ }^{6,7}$ mice $^{8}$, and dogs ${ }^{9-11}$.

The most often reported cognitive correlate of sleep spindles in humans is a positive relationship with post sleep-recall (learning success). However, this has almost exclusively been reported in smaller samples ${ }^{12-23}$, and thus the reliability of this effect requires a stronger confirmation. On one hand, the study using the largest to date sample could not find such an association ${ }^{24}$. Moreover, it is troublesome that different studies use different algorithms ${ }^{2}$, since automatic spindle detection methods diverge in their estimates of spindle occurrence ${ }^{25}$. On the other hand, invasive work in animal models has revealed putative mechanisms ${ }^{26}$ to explain how spindles promote memory consolidation, as well as implicated causality ${ }^{27}$ where human data mostly allows only for correlation. The issue thus remains controversial.

Here we report a replication analysis for the link between spindle occurrence and learning in dogs. The dog (Canis familiaris) is a fairly new model species in sleep spindle research, but one advantage in addressing the problem of replicability is that currently only one method for detecting canine spindles has been consolidated across all published studies (from one single research group, Iotchev et al. ${ }^{9-11}$ ). The same method, adopted from the human literature ${ }^{28}$ with minor alterations for use in $\operatorname{dogs}^{9}$, will also be used here to avoid the problem of automatic detector divergence ${ }^{25}$. Moreover, the current analysis will include all unpublished data sets available to us which are fit for this analysis. This is crucial because in the human literature publication bias is suspected to underlie effects reported for (fast) spindle density ${ }^{29}$. The goal of the analyses presented here will be to evaluate if the relationship between sleep spindle occurrence and learning success is real or a type I error. To evaluate this, we will look both at the prevalence of positive findings and the conditions under which a positive or negative finding is observed. We will thereby also compare the prevalence of associations between (1) single and (2) averaged measurements of sleep spindle density (spindles/minute) and learning, the latter being deemed more likely to reflect underlying traits and to be freer of measurement error.

\footnotetext{
${ }^{1}$ Department of Ethology, ELTE Eötvös Loránd University, 1117 Budapest, Hungary. ${ }^{2}$ Institute of Cognitive Neuroscience and Psychology, Research Centre for Natural Sciences, 1117 Budapest, Hungary. ${ }^{3}$ MTA-ELTE Comparative Ethology Research Group, 1117 Budapest, Hungary. ${ }^{4}$ These authors contributed equally: Ivaylo Borislavov lotchev, Vivien Reicher and Enikő Kovács. ${ }^{5}$ These authors jointly supervised this work: Anna Kis, Márta Gácsi and Enikő Kubinyi. ${ }^{\varpi}$ email: ivaylo.iotchev@gmail.com
} 


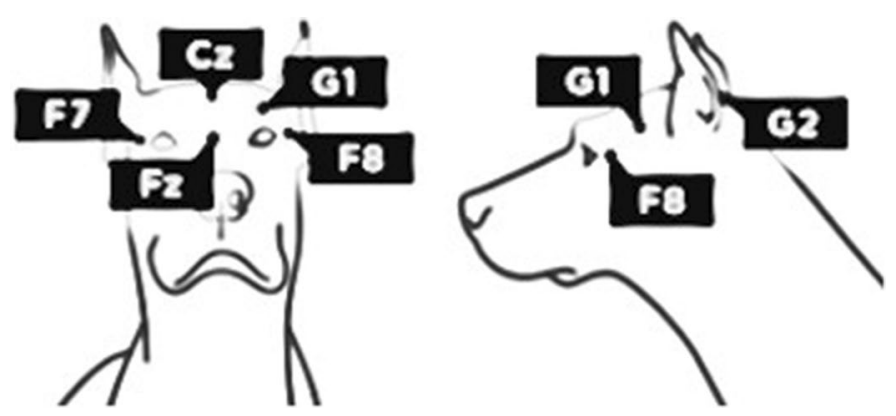

Figure 1. Schematic drawing (by Vivien Reicher) of electrode placement in the dog identical in study $1 \& 2$ (study 0 used the same electrode placement, but without the F7 channel).

\begin{abstract}
Methods
Procedure. Three datasets were included in the analyses. In all datasets the basic learning paradigm ("novel words paradigm") was based on the one used to obtain the data for Iotchev et al. ${ }^{9}$ and Kis et al. ${ }^{30}$ (for details see Supplementary Material). We included the dataset used in these studies for comparison (data set 0, N=15). Data set $1(\mathrm{~N}=19)$ originated from Reicher et al., in prep and data set $2(\mathrm{~N}=13)$ from Kovács et al. in prep. One dog participated in data set 0 and 2, otherwise the samples did not overlap. Before sleep, dogs were required to learn novel words (in English), and associate them with actions that they had been trained to perform to different verbal commands before (in Hungarian). After sleep, the final performance was measured as the percent of correct trials (out of eighteen, on the re-test), and learning gain (\% performance re-test minus test) was also calculated. All three datasets were comprised of an adaptation sleep, followed by two counter-balanced, repeated-measures conditions. Condition la used a supportive type of training (using both food and social reward in case of correct action and no scolding in case of incorrect action), while in condition $1 \mathrm{~b}$ a controlling type of training was conducted by a different experimenter (using only food reward without social reinforcement in the case of correct action and scolding in case of incorrect action). In condition 2a training was carried out by the owner in a socially relevant manner (using both food reinforcement and social reward in case of correct action), in condition $2 \mathrm{~b}$ training was carried out by an experimenter unknown to the dog in a socially irrelevant manner (using food reinforcement but without social reward in case of correct action).
\end{abstract}

Subjects. A total of 46 dogs (23 females, age range 1-9 years, 28 were purebred representatives from 16 different breeds) participated in the three studies $(0,1,2)$. One dog was included in both study 0 and 2 (a female golden retriever, aged 1 year in study 0 and 2 years in study 2). Because search for sleep spindles was restricted to non-REM sleep as in Iotchev et al. ${ }^{9,10}$, dogs which did not reach this stage or had otherwise corrupted or missing files were assigned missing values for sleep spindle density. During adaptation (occasion 1), three dogs were assigned missing values in study 0 , five dogs in study 1 , and two dogs in study 2 . Regarding data from experimental conditions (occasion 2 and 3), two missing values were assigned in condition 1a, and one in condition $2 \mathrm{a}$ (see conditions below). Missing values were excluded from our analyses and the calculation of averages.

Ethical statement. According to the Hungarian regulations of animal experimentation, our non-invasive polysomnography research does not qualify as an animal experiment. The Hungarian Scientific Ethical Committee of Animal Experiments issued a permission (under the number PE/EA/853-2/2016) approving of our noninvasive protocol. All owners volunteered to participate in the study and were informed about the procedure before beginning.

Electrode placement and EEG post-processing. Electrode placement (see Fig. 1) followed the method outlined by Kis et al. ${ }^{31}$ The polysomnographic recordings were manually categorized into sleep stages (wake, drowsiness, non-REM, REM, see Supplementary for example images) according to standard criteria $^{31}$ (validated in Gergely et al. ${ }^{32}$ ). The traces identified as non-REM (descriptive statistics in the Supplementary) were scanned for spindles using the frontal $(\mathrm{Fz})$ and central $(\mathrm{Cz})$ midline electrodes.

Spindle detection. Automatic detection was implemented as in Iotchev et al. ${ }^{9}$ on parts of the signal marked as non-REM sleep and pre-filtered between 5 and $16 \mathrm{~Hz}$. Specifics of the applied algorithm are detailed in Iotchev et al. ${ }^{9,10}$ and based on similar criteria validated against visual experts on human EEG by Nonclercq et al. ${ }^{28}$. Importantly, the algorithm invokes 2 steps, as initial detections are used to re-calculate boundaries for the target amplitude and frequency of spindles for each dog and recording, in line with the assumption that these are normally distributed within individuals. In the first step the frequency is assumed to be $9-16 \mathrm{~Hz}$ and a minimum amplitude criterion of more than 1 standard deviation above the average of the searched signal is set. For the individual adjustment in the second step the algorithm calculates maximum likelihood estimates for the means and standard deviations of amplitude and frequency. The amplitudes and frequency of the final detections have to be within 2 standard deviations of the estimated means. 
data set 0

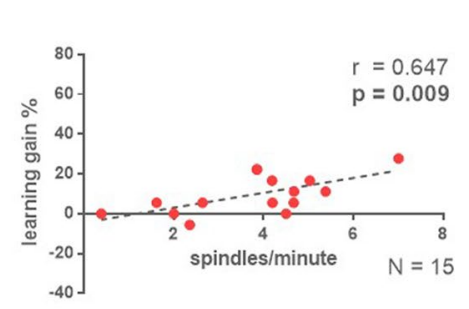

final performance

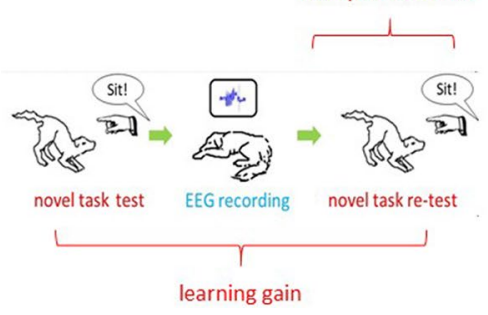

data set 1
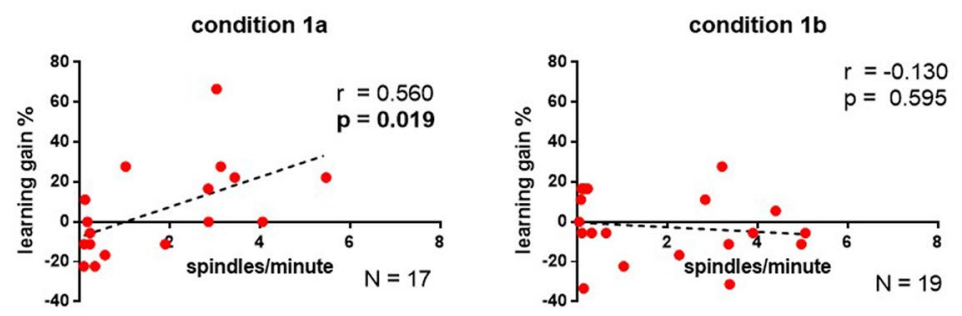

data set 2
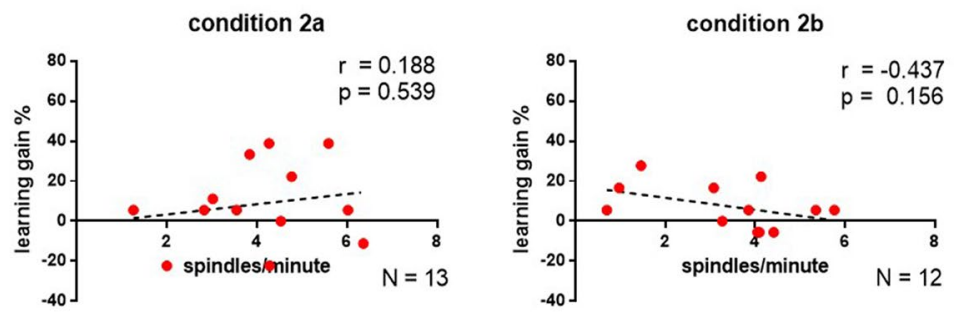

Figure 2. Associations between spindle density (spindles/minute) and learning gain as measured over the frontal midline electrode $(\mathrm{Fz})$ in data sets 0,1 , and 2 . For the already published finding in data set $0^{9}$ we used lighter colors. Two dogs did not sleep in condition $1 \mathrm{a}$ and one dog did not sleep in condition $2 \mathrm{~b}$, these animals were excluded from the analysis. Schematic drawing (by the corresponding author) of how learning gain was calculated during tests on the novel task.

Analysis. Since only Fz was active in data set 0 , we will focus below mainly on results obtained from Fz. We will also refer to and discuss detections from across the whole $9-16 \mathrm{~Hz}$ frequency range, considering that many studies looking into spindle-learning correlations did not divide spindles into slow and fast ones ${ }^{12-14,33}$. We refer to Supplementary Tables S1, S2, S4 and S5 for statistics obtained across both electrodes, and spindle sub-types (slow and fast). All associations were tested with Pearson correlations using SPSS v25.

\section{Results}

By comparing single measurements (i.e. from one single attendance, same condition) of sleep spindle occurrence and learning success, we found an association between spindle density and learning gain for data sets 0 and condition $1 \mathrm{a}$, but not for condition $1 \mathrm{~b}$ and data set 2 (see Fig. 2). Upon closer examination, both effects were specific to the slow spindle type (Table S2) and for condition 1a a trend for the same effect was observed on $\mathrm{Cz}$, as well (Table S1).

Next, we compared averaged measurements (across attendances/conditions) of sleep spindle occurrence and learning, to test how reducing measurement error could affect the relationship between spindle density and learning in the three data sets, considering that a single measurement for each variable might be less reliable ${ }^{34}$. Averaging across attendances/conditions was deemed valid, because one of the rationales for this analysis was to approximate the underlying traits, rather than estimate a condition-specific expression of either spindle occurrence or learning performance. Therefore, we averaged within each data set and for each dog spindle density values obtained from all three recordings (in data set 0 these correspond to the adaptation, control and learning conditions, while in data set 1 and 2 there was only an adaptation and two learning conditions; due to missing values, for some dogs the averages were based on only two recordings, see Supplementary for further details). We furthermore averaged learning performance variables (final performance and learning gain) for the two learning conditions in data sets 1 and 2 (this was not possible for data set 0 in which there was only one measure for each). We tested associations with both (averaged) learning gain and (averaged) final performance because their distribution and range (Table S3) suggested that for each data set a different read-out variable might better reflect the underlying learning process.

Averaged density was positively associated with learning gain in data set 0 and average final performance in data set 2 (Fig. 3). These effects were also significant for the slow sub-type and specific to Fz (Tables S4, S5). Neither averaged learning performance variables were associated with averaged density in data set 1 , but note that in condition $1 \mathrm{~b}$, more than half of the dogs (57.9\% or 11 out of 19 animals) worsened their performance on the novel task after sleep. In comparison, only 7 dogs $(36.8 \%)$ did so in condition 1a. Together with a visual inspection of the data (Fig. 1) and the performance overview provided in Table S3 (lowest average values for learning gain and final performance), these numbers suggest a floor effect on learning success in condition $1 \mathrm{~b}$.

\section{Discussion}

A positive association between dogs' spindle occurrence and learning success could be demonstrated in each of the three data sets. As a note of caution, different transformations of the raw variables (like averaged scores) and operationalization of learning (learning gain versus final performance) were used between some of the 
data set 0

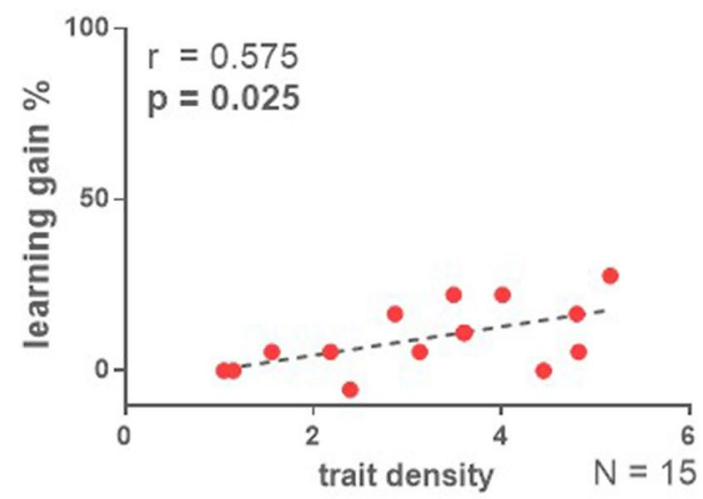

data set 2

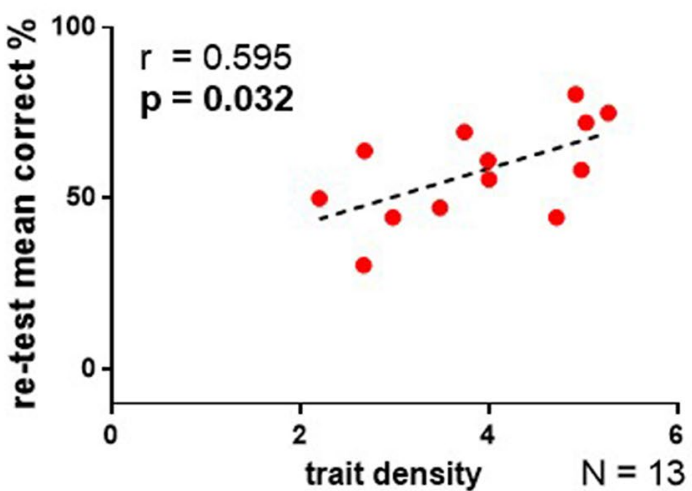

Figure 3. Associations between trait density (estimated here by averaging across recordings) and learning success (also averaged for data set 2, based on final performance) over the frontal midline electrode $(\mathrm{Fz})$ in data sets $0^{9}$ and 2. Averaging learning gain was not possible in data set 0 as the experiment consisted of only one learning condition. Data from the published data set 0 is presented in lighter colors.

comparisons, while all available samples are also small $(\mathrm{N}<20)$. An argument for treating the positive associations as cumulative derives from using the same detection algorithm ${ }^{9}$, and the same experimental operationalization of learning (the "novel words paradigm" of Kis et al. ${ }^{30}$ ) across all experiments. Intriguingly, all significant associations were specific to slow and frontal spindles (see Supplementary Tables S2, S4, S5) which resembles what is seen in humans when learning is tested with verbal material, like word-pairs ${ }^{13,16,23}$. In further support of the spindle-learning association, Type II errors are common in small, and thereby likely underpowered samples ${ }^{35}$. False negatives are also likely, considering that memory consolidation is not restricted to sleep alone in neither humans nor $\operatorname{dogs}^{30,36}$. Also, many additional conditions are known to influence if any effect is observed, e.g. relative timing to ripples and slow-waves ${ }^{37}$, emotional arousal ${ }^{38}$ and exact stage of non-REM sleep ${ }^{14}$. However, since in most animals it is hard to separate non-REM sleep stages from each other ${ }^{31,39}$ not all of these conditions can be tested outside of humans.

Surprisingly, although sleep-dependent memory consolidation operates in the time-frame of a single $\mathrm{day}^{40,41}$ and exposure to new information has been shown to result in direct increases in spindle occurrence in humans ${ }^{15,23}$, rats $^{33}$ and dogs ${ }^{9}$, our results for data sets 0 and 2 suggest that estimating trait density by averaging across recordings might increase the visibility of spindle-learning associations. Other arguments for the predictive utility of trait density come from reports of stable spindle occurrence across nights in humans ${ }^{42,43}$, the heritability of sleep spindle density ${ }^{44}$, and the observation that different psychiatric conditions and natural aging, each associated with memory problems, can measurably reduce spindle occurrence in humans ${ }^{45-49}$ and specifically the occurrence of slow spindles in $\operatorname{dog} s^{10}$.

We conclude that the here examined data-sets lend additional support to the positive association between sleep spindle occurrence and learning observed in dogs earlier ${ }^{9}$, but the need for further evidence is not exhausted. Even more and larger samples will be required to establish to what extend low power accounts for the proportion of null results. Moreover, since trait occurrence is also associated with general mental ability ${ }^{50}$ more studies with a control condition, in which sleep is not preceded by learning demand, will be needed in dogs to separate if these correlations reflect memory consolidation or general learning potential.

Received: 19 June 2020; Accepted: 17 December 2020

Published online: 31 December 2020

\section{References}

1. Steriade, M. \& Llinás, R. R. The functional states of the thalamus and the associated neuronal interplay. Physiol. Rev. 68, 649-742 (1988).

2. Fernandez, L. M. J. \& Lüthi, A. Sleep spindles: Mechanisms and functions. Physiol. Rev. https://doi.org/10.1152/physrev.00042 $.2018(2020)$.

3. Kryger, M. H., Roth, T. \& Dement, W. C. Principles and Practice of Sleep Medicine (Saunders/Elsevier, Amsterdam, 2011).

4. Rechtschaffen, A. \& Kales, A. A Manual of Standardized Techniques and Scoring System for Sleep Stages of Human Subjects (D.C. U.S. Gov. Print. Off. NIH Public, Washington, 1968).

5. Dutertre, F. Catalog of the Main EEG-Patterns. Handbook of Electroencephalography and Clinical Neurophysiology (Elsevier, Amsterdam, 1977).

6. Bódizs, R., Körmendi, J., Rigó, P. \& Lázár, A. S. The individual adjustment method of sleep spindle analysis: Methodological improvements and roots in the fingerprint paradigm. J. Neurosci. Methods 178, 205-213 (2009).

7. De Gennaro, L. et al. The electroencephalographic fingerprint of sleep is genetically determined: A twin study. Ann. Neurol. 64, 455-460 (2008).

8. Kim, D., Hwang, E., Lee, M., Sung, H. \& Choi, J. H. Characterization of topographically specific sleep spindles in mice. Sleep 38, 85-96 (2015).

9. Iotchev, I. B., Kis, A., Bódizs, R., van Luijtelaar, G. \& Kubinyi, E. EEG transients in the sigma range during non-REM sleep predict learning in dogs. Sci. Rep. 7, 12936 (2017). 
10. Iotchev, I. B. et al. Age-related differences and sexual dimorphism in canine sleep spindles. Sci. Rep. 9, 10092 (2019).

11. Iotchev, I. B., Szabó, D., Kis, A. \& Kubinyi, E. Possible association between spindle frequency and reversal-learning in aged family dogs. Sci. Rep. 10, 6505 (2020).

12. Clemens, Z., Fabó, D. \& Halász, P. Twenty-four hours retention of visuospatial memory correlates with the number of parietal sleep spindles. Neurosci. Lett. 403, 52-56 (2006).

13. Clemens, Z., Fabó, D. \& Halász, P. Overnight verbal memory retention correlates with the number of sleep spindles. Neuroscience 132, 529-535 (2005).

14. Cox, R., Hofman, W. F. \& Talamini, L. M. Involvement of spindles in memory consolidation is slow wave sleep-specific. Learn. Mem. 19, 264-267 (2012).

15. Gais, S., Mölle, M., Helms, K. \& Born, J. Learning-dependent increases in sleep spindle density. J. Neurosci. 22, 6830-6834 (2002).

16. Kuula, L. et al. Higher sleep spindle activity is associated with fewer false memories in adolescent girls. Neurobiol. Learn. Mem. 157, 96-105 (2019).

17. Seeck-Hirschner, M. et al. Declarative memory performance is associated with the number of sleep spindles in elderly women. Am. J. Geriatr. Psychiatry 20, 782-788 (2012).

18. Lustenberger, C. et al. Feedback-controlled transcranial alternating current stimulation reveals a functional role of sleep spindles in motor memory consolidation. Curr. Biol. 26, 2127-2136 (2016).

19. Yordanova, J., Kolev, V., Bruns, E., Kirov, R. \& Verleger, R. Sleep spindles in the right hemisphere support awareness of regularities and reflect pre-sleep activations. Sleep 40, 1-13 (2017).

20. Barakat, M. et al. Fast and slow spindle involvement in the consolidation of a new motor sequence. Behav. Brain Res. 217, 117-121 (2011).

21. Tamaki, M., Matsuoka, T., Nittono, H. \& Hori, T. Activation of fast sleep spindles at the premotor cortex and parietal areas contributes to motor learning: A study using sLORETA. Clin. Neurophysiol. 120, 878-886 (2009).

22. Astill, R. G. et al. Sleep spindle and slow wave frequency reflect motor skill performance in primary school-age children. Front. Hum. Neurosci. https://doi.org/10.3389/fnhum.2014.00910 (2014).

23. Schmidt, C. et al. Encoding difficulty promotes postlearning changes in sleep spindle activity during napping. J. Neurosci. 26, 8976-8982 (2006).

24. Ackermann, S., Hartmann, F., Papassotiropoulos, A., de Quervain, D. J. F. \& Rasch, B. No associations between interindividual differences in sleep parameters and episodic memory consolidation. Sleep 38, 951-959 (2015).

25. Warby, S. C. et al. Sleep-spindle detection: Crowdsourcing and evaluating performance of experts, non-experts and automated methods. Nat. Methods 11, 385-392 (2014).

26. Rosanova, M. \& Ulrich, D. Pattern-specific associative long-term potentiation induced by a sleep spindle-related spike train. J. Neurosci. 25, 9398-9405 (2005).

27. Latchoumane, C. F. V., Ngo, H. V. V., Born, J. \& Shin, H. S. Thalamic spindles promote memory formation during sleep through triple phase-locking of cortical, thalamic, and hippocampal rhythms. Neuron 95, 424-435.e6 (2017).

28. Nonclercq, A. et al. Sleep spindle detection through amplitude-frequency normal modelling. J. Neurosci. Methods 214, 192-203 (2013).

29. Ujma, P. P. Sleep spindles and general cognitive ability-A meta-analysis. Sleep Spindl. Cortical Up States. https://doi. org/10.1556/2053.2.2018.01 (2018).

30. Kis, A. et al. The interrelated effect of sleep and learning in dogs (Canis familiaris); an EEG and behavioural study. Sci. Rep. 7, 41873 (2017).

31. Kis, A. et al. Development of a non-invasive polysomnography technique for dogs (Canis familiaris). Physiol. Behav. 130, 149-156 (2014).

32. Gergely, A. et al. Reliability of family dogs' sleep structure scoring based on manual and automated sleep stage identification. Animals. https://doi.org/10.3390/ani10060927 (2020).

33. Eschenko, O., Molle, M., Born, J. \& Sara, S. J. Elevated sleep spindle density after learning or after retrieval in rats. J. Neurosci. 26, 12914-12920 (2006).

34. Reynolds, C. M., Gradisar, M. \& Short, M. A. Reliability of sleep spindle measurements in adolescents: How many nights are necessary?. J. Sleep Res. https://doi.org/10.1111/jsr.12698 (2019).

35. Schmidt, F. L. \& Hunter, J. E. Methods of Meta-Analysis: Correcting Error and Bias in Research Findings (SAGE Publications, Thousand Oaks, 2004).

36. Korman, M. et al. Daytime sleep condenses the time course of motor memory consolidation. Nat. Neurosci. 10, 1206-1213 (2007).

37. Clemens, Z. et al. Fine-tuned coupling between human parahippocampal ripples and sleep spindles. Eur. J. Neurosci. 33, 511-520 (2011).

38. Lehmann, M., Schreiner, T., Seifritz, E. \& Rasch, B. Emotional arousal modulates oscillatory correlates of targeted memory reactivation during NREM, but not REM sleep. Sci. Rep. https://doi.org/10.1038/srep39229 (2016).

39. Genzel, L., Kroes, M. C. W., Dresler, M. \& Battaglia, F. P. Light sleep versus slow wave sleep in memory consolidation: A question of global versus local processes?. Trends Neurosci. 37, 10-19 (2014).

40. Stickgold, R., James, L. \& Hobson, J. Visual discrimination learning requires sleep after training. Nat. Neurosci. 3, 1237 (2000).

41. Tse, D. et al. Schemas and memory consolidation. Science 316, 76-82 (2007).

42. Silverstein, L. D. \& Levy, C. M. The stability of the sigma sleep spindle. Electroencephalogr. Clin. Neurophysiol. 40, 666-670 (1976).

43. Gaillard, J. M. \& Blois, R. Spindle density in sleep of normal subjects. Sleep 4, 385-391 (1981).

44. Hori, A. Sleep characteristics in twins. Psychiatry Clin. Neurosci. 40, 35-46 (1986).

45. Gorgoni, M. et al. Parietal fast sleep spindle density decrease in Alzheimer's disease and amnesic mild cognitive impairment. Neural Plast. 2016, 10 (2016).

46. Ferrarelli, F. et al. Reduced sleep spindle activity in schizophrenia patients. Am. J. Psychiatry 164, 483-492 (2007).

47. Merikanto, I. et al. ADHD symptoms are associated with decreased activity of fast sleep spindles and poorer procedural overnight learning during adolescence. Neurobiol. Learn. Mem. 157, 106-113 (2019).

48. Guazzelli, N. et al. Sleep spindles in normal elderly: Comparison with young adult patterns and relation to nocturnal awakening, cognitive function and brain atrophy. Electroencephalogr. Clin. Neurophysiol. 63, 526-539 (1986).

49. Smirne, S. et al. Sleep in presenile dementia. Electroencephalogr. Clin. Neurophysiol. 43, 4 (1977)

50. Fogel, S. M., Nader, R., Cote, K. A. \& Smith, C. T. Sleep spindles and learning potential. Behav. Neurosci. 121, 1-10 (2007).

\section{Acknowledgements}

We thank our dog trainers Rita Báji and Barbara Csibra for their contributions to the work behind data set 1, as well as all owner-dog pairs who participated in the discussed studies; Borbála Turcsan, Tamás Faragó for useful comments to this manuscript, and Péter Ujma for inspiring debates on the reliability of spindle-learning correlations. 


\section{Author contributions}

I.I. wrote the detection algorithm, analyzed the data and wrote the initial draft; R.V., A.K., E.Ko. and T.K. collected data and participated in the writing, A.K., M.G. and E.Ku. supervised the research, participated in planning the experiments and in the writing process.

\section{Funding}

This work has received funding from the European Research Council (ERC) under the European Union's Horizon 2020 research and innovation programme (Grant Agreement No. 680040), the National Research Development and Innovation Office (OTKA FK128242, K132372), the BIAL Foundation (Grant No 169/16), Hungarian Academy of Sciences (F01/031) and the János Bolyai Research Scholarship of the Hungarian Academy of Sciences.

\section{Competing interests}

The authors declare no competing interests.

\section{Additional information}

Supplementary Information The online version contains supplementary material available at https://doi. org/10.1038/s41598-020-80417-8.

Correspondence and requests for materials should be addressed to I.B.I.

Reprints and permissions information is available at www.nature.com/reprints.

Publisher's note Springer Nature remains neutral with regard to jurisdictional claims in published maps and institutional affiliations.

(c) (i) Open Access This article is licensed under a Creative Commons Attribution 4.0 International License, which permits use, sharing, adaptation, distribution and reproduction in any medium or format, as long as you give appropriate credit to the original author(s) and the source, provide a link to the Creative Commons licence, and indicate if changes were made. The images or other third party material in this article are included in the article's Creative Commons licence, unless indicated otherwise in a credit line to the material. If material is not included in the article's Creative Commons licence and your intended use is not permitted by statutory regulation or exceeds the permitted use, you will need to obtain permission directly from the copyright holder. To view a copy of this licence, visit http://creativecommons.org/licenses/by/4.0/.

(c) The Author(s) 2020 